\title{
Commentary on Paper "Drug-Induced HSP90 Inhibition Alleviates Pain in Monoarthritic Rats and Alters the Expression of New Putative Pain Players at the DRG"

\author{
Fani L. Moreira Neto ${ }^{1,2,3 *}$
}

'Instituto de Investigação e Inovação em Saúde, Universidade do Porto, Rua Alfredo Allen 208, 4200-393 Porto, Portugal. ${ }^{2}$ IBMC - Instituto de Biologia Molecular e Celular, Universidade do Porto, Rua Alfredo Allen 208, 4200-393 Porto, Portugal. ${ }^{3}$ Departamento de Biomedicina - Unidade de Biologia Experimental, Faculdade de Medicina, Universidade do Porto, Alameda Prof Hernâni Monteiro, $4200-319$ Porto, Portugal.

Article Info

\section{Article Notes}

Received: August 10, 2018

Accepted: October12, 2018

\section{*Correspondence:}

Dr. Fani L. Moreira Neto, Departamento de Biomedicina Unidade de Biologia Experimental, Faculdade de Medicina da Universidade do Porto (FMUP), Alameda Prof Hernâni Monteiro, 4200-319 Porto, Portugal; Telephone No: +351 22 0426769; Fax No: +351 22 5513655;

E-mail: fanineto@med.up.pt.

(C) 2018 Neto FLM. This article is distributed under the terms of the Creative Commons Attribution 4.0 International License.

\section{Keywords:}

Heat Shock Protein 90 (HSP90)

Dorsal Root Ganglia (DRG)

Monoarthritis

Satellite Glial Cells (SGC).

\section{ABSTRACT}

Heat shock protein 90 (HSP90) belongs to a highly conserved family of molecular chaperones and is responsible for regulating the protein folding quality control of specific client proteins. In a recent study published in Molecular Neurobiology, HSP90mRNA levels were found significantly decreased after knock-down in vitro of activating transcription factor 3 (ATF3), indicating that this stress-inducible gene that mediates pro-apoptosis or cytoprotection might act as positive regulator of HSP90 expression. In the rodent model of Monoarthritis, characterized by being accompanied by chronic joint inflammatory pain, the mRNA and protein levels for HSP9O were significantly increased in dorsal root ganglia (DRG). Additionally, a reversal in the HSP90 mRNA upregulation and in the 70kDa protein isoform levels following intrathecal delivery of a HSP90 inhibitor, along with an attenuation of movement-induced mechanical allodynia, and reduced neuronal sensitization and satellite glial cells (SGC) activation in ipsilateral DRG of the arthritic animals were also observed. This suggests a putative role of HSP90 in chronic inflammatory pain pathophysiology at sensory ganglia level that is still unexplored. To date only a few studies demonstrated a link between pain and HSP90 modulation, but there are several evidences that HSP90 is involved in inflammation, tumorigenesis and neurodegeneration. Here, we discuss the status of the studies demonstrating a role for HSP9O in inflammation and comment on their possible involvement in neuronal/glial driven pain mechanisms.

\section{Contextualization}

Heat shock proteins (HSPs) are induced in response to a wide variety of insults including temperature, stress, hypoxia, inflammation or infections. Working as sensors of stress, they contribute to cell survival in adverse conditions. Amongst many cytoprotective mechanisms, they are involved in protein identification and degradation (by addressing them to the proteasome) as well as the regulation of apoptotic machineries at the pre- and postmitochondrial level ${ }^{1,2}$. Importantly, as chaperones, they also have a prominent role in physiological states by controlling the correct folding of newly synthesized polypeptides, assembly of protein complexes and protein transport ${ }^{3}$. Due to the referred chaperone and anti-apoptotic properties, HSPs have been gaining relevance in numerous pathologies, namely in several types of cancer. Indeed, they are overexpressed in breast tumours, lung cancer, leukemia and Hodgkin lymphoma, contributing to 
tumorigenicity and cancer cell resistance ${ }^{4}$. More recently, HSPs' implication in neurodegenerative diseases, mostly associated with protein misfolding or aberrant formation of aggregates, has been drawing the researcher's attention and leading to effective therapies ${ }^{5-8}$. However, regardless of the advances in the last decade, the HSPs' role in neurons and how survival is promoted in these cells is still practically unknown.

Our studies have been focusing on the neurobiological changes occurring at the sensory neurons upon painful joint inflammation. We have previously demonstrated that one of the features of the Monoarthritis model of chronic inflammatory joint pain is the induction of activating transcriptional factor 3 (ATF3) in dorsal root ganglia (DRG) neurons at early stages of the disease ${ }^{9}$. Quite surprisingly, we observed a significant decrease in the mRNA expression of the HSP90 chaperone when silencing ATF3 expression in vitro, indicating that this neuronal injury marker acts as positive regulator of HSP90 expression ${ }^{10}$. ATF3 is a stressinducible gene that, depending on the environmental cues, is capable of mediating pro-apoptosis or promoting cell survival ${ }^{11-13}$, and therefore the association between this factor and HSP90 was quite interesting. These findings further motivated us to evaluate HSP90 role in Monoarthritis and possibly in nociception, which later resulted in a publication in Molecular Neurobiology ${ }^{10}$. Apart from our study, to date only a few studies demonstrated a link between pain and HSP90 modulation ${ }^{14-17}$. In the May 2018 issue of Molecular Neurobiology, we show that HSP90 expression is increased in the DRG of monoarthritic animals and we proposed that ATF3 alert signals in inflammation could be mediated by/with this chaperone. In fact, the administration of an HSP90 inhibitor during Monoarthritis resulted in several molecular alterations including the reversion of HSP90 overexpression, as well as down-regulation of the neuronal purinergic receptor P2X3 (highly correlated to pain ${ }^{18}$ ) and of glial fibrillary acidic protein (a marker of glial cells activation, which are also greatly involved in pain ${ }^{19}$ ). These changes were accompanied by a reduction in mechanical hypersensitivity thus revealing a functional outcome associated with HSP90 inhibition.

In spite of these findings, many questions still remain. Indeed, evaluating the biological significance of these events and tackling the exact mechanisms by which these changes occur is a complex task. Certainly, gathering the pieces of this puzzle together and dissecting HSP90mediated signaling pathways in neurons will be of great clinical relevance for several diseases. This might be of special interest for the management of pain during inflammation since common analgesic treatments are so often ineffective. Here, we will further discuss the status of the studies demonstrating a role for HSP90 in inflammation and comment on their possible involvement in neuronal/ glial driven pain mechanisms.

\section{HSP90 Inhibition: Potential as An Anti-Inflammatory Strategy}

There are two isoforms of the HSP90 chaperone, the beta, which is constitutively expressed, and the alpha that is inducible upon stimulus. They both function in an ATP-dependent manner being essential for the viability of eukaryotic cells. They form chaperone complexes with other relevant proteins in order to regulate the folding, activation, and assembly of numerous client proteins, including key mediators of signal transduction and transcriptional factors. Concerning its anti-apoptotic role, HSP90 also interacts and stabilizes the receptor interacting kinase (RIP) which, upon stimulation by Tumour Necrosis Factor alpha (TNF- $\alpha$ ), culminates in the activation of Nuclear Factor-kappa B (NF- $\kappa \mathrm{B})$. Thus, inactivation of HSP90 leads to RIP degradation and prevents the activation of $\mathrm{NF}-\mathrm{kB}$, thereby resulting in the suppression of a key component of the inflammatory response ${ }^{20}$. The list of HSP90 client proteins includes also the JAK/Signal Transducer and Activator of Transcription (STATs) (JAK2) and Toll Like-Receptor 4 (TLR-4), which are both also important regulators of signaling pathways that mediate inflammatory and immune responses ${ }^{2}$. Indeed, most of the HSP90 inhibitors act by interrupting the intrinsic ATPase activity, causing instead the degradation of the client proteins via the ubiquitin-proteasome pathway. HSP90 inhibition also triggers the activation of heat shock factor-1 (HSF-1), resulting in an increased expression of factors, such as IL-10 and Hsp70, that suppress pro-inflammatory and activate anti-inflammatory genes. Additionally, studies on autoimmune/inflammatory diseases indicate that the HSP90 inhibitors action is associated with the selective blockade of innate and adaptive cells of the immune system $^{21}$. Thus, since many key regulators in inflammation and immune responses are HSP90 clients, the rationale for the reported efficacy of HSP90 inhibitors as antiinflammatory drugs is quite comprehensible.

The first class of HSP90 inhibitors in clinical trials includes drugs as a geldanamycin analog, 17-allylamino, 17-demethoxygeldanamycin (17AAG) and 17-Dimethylaminoethylamino-17demethoxygeldanamycin (17-DMAG) ${ }^{22-24}$. Besides having been extensively explored in the tumor context, these drugs were shown to reduce cytokines and nitric oxide (NO) production and to attenuate the inflammatory response either in encephalomyelitis ${ }^{25}$, severe sepsis ${ }^{26}$ or experimental stroke ${ }^{27}$. However, for the purposes of our studies, it was even more interesting to find that similar results were exerted in rheumatoid arthritis ${ }^{28}$, suggesting an effect both at the level of innate and adaptive immune system. In our hands, the administration of 17- 
DMAG to monoarthritic animals reversed HSP90 mRNA upregulation and attenuated the movement-induced mechanical allodynia in the first hours post-administration. These findings suggest a putative role of HSP90 in the pathophysiology of chronic inflammatory pain at sensory ganglia level that is still completely unexplored ${ }^{10}$. Interestingly, while trying to decipher the underlying mechanisms, we were also able to detect a significant increase in the DRG of inflamed animals of a smaller form of HSP90, with around $70 \mathrm{kDa}$, that our experiments suggested to be a cleaved form of the chaperone. In fact, it had previously been shown that reactive oxygen species (ROS), which are normally found elevated in inflammatory conditions like monoarthritis, are responsible for HSP90 non-enzymatic cleavage ${ }^{29}$. However, even though this is a plausible mechanistic explanation, the possibility of a nonenzymatic cleavage still remains to be confirmed, namely by the use of ROS scavengers, while other events might also justify the appearance of this form. Indeed, besides the cytoplasmic HSP90 $\alpha$ and HSP90 $\beta$ isoforms, there are several other isoforms of HSP90 that may exist compartmentalized in discrete subcellular microenvironments and organelles. GRP94 (Glucose-regulated protein 94), for instance, is the HSP90-like protein located in the lumen of the endoplasmic reticulum while the mitochondrial-compartmentalized Hsp90-like chaperone is known as Tumor Necrosis Factor Receptor-Associated Protein 1 (TRAP-1), a protein of about $\sim 75 \mathrm{kDa}$, initially named Hsp75 ${ }^{30,31}$. There is evidence that chaperone control of proteostasis is often altered in pathological conditions and that organelle-specific HSP90 molecules act as cores of several cellular signalling pathways regulating homeostasis. Interestingly, TRAP-1 is involved in protecting mitochondrial integrity from ROS, defending against mitochondrial apoptosis, thereby helping cell survival. In tumor cells, TRAP-1 appears to be crucial in regulating mitochondrial bioenergetics, significantly influencing neoplastic growth ${ }^{32}$. Moreover, there is now evidence that deregulation of the TRAP-1 cytoprotection pathways is implicated in neurodegeneration ${ }^{30,33}$. Taking this into consideration, we do not exclude that mitochondrial forms of HSP90 play a role in Monoarthritis, as we discuss below, and that, besides the suppression of typical inflammatory signaling cascades, the analgesic effects of HSP90 inhibition that we observed might also be explained by neuroprotective events. The use of newly developed specific TRAP-1 inhibitors ${ }^{34,35}$ will be helpful to ascertain this.

\section{Role for HSP90 in Pain - New Evidence}

It seems plausible that HSP90 might exert analgesic effects either indirectly by the suppression of inflammatory cascades or more directly by promoting neuronal protection and survival. Indeed, by regulating the expression of transcriptional factors (as NF- $\mathrm{KB}$ ) and the production of pro-inflammatory molecules, HSP90 can totally modulate the inflammatory response, which will probably have an impact on nociception. In our studies, upon HSP90 inhibition by 17-DMAG administration to monoarthritic animals there was an attenuation of satellite glial cells (SGCs) activation in sensory ganglia. These events are most likely correlated to the observed reduced hypersensitivity. In fact, even though the levels of inflammatory mediators were not assessed in our experiments, a reduced release is likely to have occurred, as HSP90 inhibition is associated with an anti-inflammatory effect. Moreover, the activation of SGCs has long been associated with inflammatory responses and pain processing ${ }^{36}$ and their inhibition alleviates pain ${ }^{7}$.

Also in support of a role of HSP90 in allodynia, others have shown that intrathecal administration of the HSP90 inhibitor 17-DMAG alleviated neuropathic pain via a TLR4 pathway ${ }^{14,37}$. Indeed, TLR4 activation by exogenous ligands (curiously amongst them HSP90) triggers typical inflammatory signaling cascades and is therefore highly correlated to both inflammatory and neuropathic pain ${ }^{38,39}$. Moreover, the intracellular TLR4 cascade has been associated not only with HSP90 activity but also with other relevant chaperones like HSP70 and transcriptional factors as ATF3 ${ }^{12,14,40}$. As mentioned above, ATF3 seems to be a key regulator in Monoarthritis, orchestrating the shift of initial inflammatory mechanisms to later events with a more neuropathic phenotype that occur during disease progression. It is currently accepted that neuronal injury or neuroinflammation are not limited to detrimental events but these go hands-with-hands with the induction of rescuing mechanisms. Thus, it is conceivable that ATF3 cooperates with HSPs in stress signaling during joint inflammatory conditions and that these events are implicated somehow with pain processing as a mechanism of tissue protection. Accordingly, we found that ATF3 knock-down resulted in significant decreases of HSP90 in cultured sensory neurons, while HSP90 inhibition in MA animals contributed to great increases in ATF3 expression ${ }^{10}$.

Besides the impact in the distinct components of the inflammatory response, some of the strongest hints for a role for HSPs in pain neurobiology arise from studies in neurodegeneration and neuroprotection. Accordingly, a deleterious effect of HSP90 in neurodegeneration has been proposed as it may maintain the functional stability of neuronal proteins of aberrant capacity, like those that accumulate in neurodegenerative diseases ${ }^{6,8}$. Is it then possible that the HSP90 action may also support the integrity and function of a number of immune mediators, neuropeptides and receptors that are intensively produced by sensory neurons in conditions of peripheral nerve injury and/or neuroinflammation, contributing therefore to the neuronal sensitization that occurs in 
painful conditions? In fact, it is noteworthy that, upon HSP90 inhibition, we found decreased levels of P2X3 in sensory neurons of monoarthritic animals (and therefore diminished neuronal sensitization) along with decreased mechanical allodynia ${ }^{10}$. However, these anti-nociceptive effects derived from HSP90 inhibition by 17-DMAG cannot directly associate with a restitution of the chaperone functioning. Although cleavage was prevented, the drug acts by blocking HSP90 ATPase activity and therefore the protein remains most likely dysfunctional in the treated animals. Thus, we proposed that 17-DMAG effects might be mediated by HSF1. In unstressed cells HSF1 is inactive forming a complex with HSP90 but when 17-DMAG binds to HSP90, similarly to what happens in stressful conditions, HSF1 dissociates and translocates to the nucleus, promoting the expression of stress-responsive genes ${ }^{41-44}$. This mechanism could possibly explain the molecular changes observed following 17-DMAG treatment, even though HSP90 is probably not functional. Indeed, many of the neuroprotective actions described for HSP90 inhibitors were shown to be exerted by activation and translocation of HSF-1, which results in increased expression of HSP70 and HSP40. HSP70 molecular chaperones attenuate both the intrinsic and extrinsic apoptotic pathways, promoting cell proliferation and survival both by acting as co-chaperones of HSP90 and by neutralizing the effects of a number of proapoptotic proteins ${ }^{45}$. Curiously, also the co-expression of HSP70 and ATF3 has already been observed in a subset of motoneurons in a transgenic model of amyotrophic lateral sclerosis, a very aggressive neurodegenerative disease $\mathrm{e}^{46}$. Thus, although there is lack of studies to prove this, it is equally possible that the effects of HSP90 inhibition on the attenuation of nociception and neuronal sensitization might occur through HSF-1 and HSP70-mediated events ${ }^{6,8}$. Lastly, there is evidence that in diabetic painful neuropathy rodent models, where the neuronal mitochondrial function is decreased, HSP90 inhibitors improved thermal hypoalgesia and enhanced mitochondrial bioenergetics in sensory neurons ${ }^{16,17,47}$. Thus, it is also plausible that HSP90 inhibition effects on nociception might be related with the prevention of mitochondrial dysfunction in sensory neurons. Curiously, in our studies we have detected a $70 \mathrm{KDa}$ isoform of HSP90 with increased expression during Monoarthritis, that could represent the mitochondrial isoform ${ }^{10}$. Additionally, as ROS production results in great part from mitochondria dysfunction and NADPH oxidases (NOXs) signaling dysregulation ${ }^{48,49}$ and TRAP1 increased the basal ROS levels and decreased mitochondrial biogenesis in mouse fibroblast cells ${ }^{50}$, it is also possible that the ROS-mediated HSP90 cleavage might result from TRAP1 signaling dysregulation. A set of new experiments are planned to explore this possibility.

In conclusion, data point to a role of HSP90 in the processing of the nociceptive information, possibly by regulating the proteostasis of several players leading to neuroinflammation, neuronal sensitization and/or neurodegeneration. However, the underlying mechanisms are still unknown. The delivery of HSP90 inhibitors yields antinociceptive effects, at least during some time after administration. Therefore, as in cancer therapy, in which HSP90 inhibitors seem to be promising, it might be of great relevance to better understand these mechanisms in order to develop more tailored pharmacological tools to target the periphery and alleviate chronic pain.

\section{References}

1. Didelot C, Schmitt E, Brunet M, et al. Heat shock proteins: endogenous modulators of apoptotic cell death. Handb Exp Pharmacol. 2006; (172): 171-98.

2. Sevin M, Girodon F, Garrido C, et al. HSP90 and HSP70: Implication in Inflammation Processes and Therapeutic Approaches for Myeloproliferative Neoplasms. Mediators Inflamm. 2015; 2015: 970242.

3. Jindal S. Heat shock proteins: applications in health and disease. Trends Biotechnol. 1996; 14(1): 17-20.

4. Calderwood SK. Heat shock proteins and cancer: intracellular chaperones or extracellular signalling ligands? Philos Trans R Soc Lond B Biol Sci. 2018; 373(1738).

5. Adachi $\mathrm{H}$, Katsuno $\mathrm{M}$, Waza $\mathrm{M}$, et al. Heat shock proteins in neurodegenerative diseases: pathogenic roles and therapeutic implications. Int J Hyperthermia. 2009; 25(8): 647-54.

6. Lackie RE, Maciejewski A, Ostapchenko VG, et al. The Hsp70/Hsp90 Chaperone Machinery in Neurodegenerative Diseases. Front Neurosci. 2017; $11: 254$

7. Liu FY, Sun YN, Wang FT, et al. Activation of satellite glial cells in lumbar dorsal root ganglia contributes to neuropathic pain after spinal nerve ligation. Brain Res. 2012; 1427: 65-77.

8. Luo $\mathrm{W}$, Sun $\mathrm{W}$, Taldone $\mathrm{T}$, et al. Heat shock protein 90 in neurodegenerative diseases. Mol Neurodegener. 2010; 5: 24.

9. Nascimento D, Pozza DH, Castro-Lopes JM, et al. Neuronal injury marker ATF-3 is induced in primary afferent neurons of monoarthritic rats. Neurosignals. 2011; 19(4): 210-21.

10. Nascimento DSM, Potes CS, Soares ML, et al. Drug-Induced HSP90 Inhibition Alleviates Pain in Monoarthritic Rats and Alters the Expression of New Putative Pain Players at the DRG. Mol Neurobiol. 2018; 55(5): 3959-3975.

11. Gilchrist M, Henderson WR, Jr., Morotti A, et al. A key role for ATF3 in regulating mast cell survival and mediator release. Blood. 2010; 115(23): 4734-41.

12. Hunt D, Raivich G, and Anderson PN. Activating transcription factor 3 and the nervous system. Front Mol Neurosci. 2012; 5: 7.

13. Yang H, Park SH, Choi HJ, et al. Epithelial cell survival by activating transcription factor 3 (ATF3) in response to chemical ribosomeinactivating stress. Biochem Pharmacol. 2009; 77(6): 1105-15.

14. Hutchinson MR, Ramos KM, Loram LC, et al. Evidence for a role of heat shock protein-90 in toll like receptor 4 mediated pain enhancement in rats. Neuroscience. 2009; 164(4): 1821-32.

15. Lei W, Mullen N, McCarthy S, et al. Heat-shock protein 90 (Hsp90) promotes opioid-induced anti-nociception by an ERK mitogenactivated protein kinase (MAPK) mechanism in mouse brain. J Biol Chem. 2017; 292(25): 10414-10428.

16. Urban MJ, Li C, Yu C, et al. Inhibiting heat-shock protein 90 reverses sensory hypoalgesia in diabetic mice. ASN Neuro. 2010; 2(4): e00040. 
17. Urban MJ, Pan P, Farmer KL, et al. Modulating molecular chaperones improves sensory fiber recovery and mitochondrial function in diabetic peripheral neuropathy. Exp Neurol. 2012; 235(1): 388-96.

18. North RA. P2X3 receptors and peripheral pain mechanisms. J Physiol. 2004; 554(Pt 2): 301-8.

19. Ji RR, Berta T, and Nedergaard M. Glia and pain: is chronic pain a gliopathy? Pain. 2013; 154 Suppl 1: S10-28.

20. Zhang $\mathrm{H}$ and Burrows $\mathrm{F}$. Targeting multiple signal transduction pathways through inhibition of Hsp90. J Mol Med (Berl). 2004; 82(8): 488-99.

21. Tukaj S and Wegrzyn G. Anti-Hsp90 therapy in autoimmune and inflammatory diseases: a review of preclinical studies. Cell Stress Chaperones. 2016; 21(2): 213-8.

22. Aregbe AO, Sherer EA, Egorin MJ, et al. Population pharmacokinetic analysis of 17-dimethylaminoethylamino-17demethoxygeldanamycin (17-DMAG) in adult patients with solid tumors. Cancer Chemother Pharmacol. 2012; 70(1): 201-5.

23. Hao H, Naomoto Y, Bao X, et al. HSP90 and its inhibitors. Oncol Rep. 2010; 23(6): 1483-92.

24. Sharp S and Workman P, Inhibitors of the HSP90 molecular chaperone: current status. Adv Cancer Res. 2006; 95: 323-48.

25. Dello Russo C, Polak PE, Mercado PR, et al. The heat-shock protein 90 inhibitor 17-allylamino-17-demethoxygeldanamycin suppresses glial inflammatory responses and ameliorates experimental autoimmune encephalomyelitis. J Neurochem. 2006; 99(5): 1351-62.

26. Chatterjee A, Dimitropoulou C, Drakopanayiotakis F, et al. Heat shock protein 90 inhibitors prolong survival, attenuate inflammation, and reduce lung injury in murine sepsis. Am J Respir Crit Care Med. 2007; 176(7): 667-75.

27. Qi J, Han X, Liu HT, et al. 17-Dimethylaminoethylamino-17demethoxygeldanamycin attenuates inflammatory responses in experimental stroke. Biol Pharm Bull. 2014; 37(11): 1713-8.

28. Rice JW, Veal JM, Fadden RP, et al. Small molecule inhibitors of Hsp90 potently affect inflammatory disease pathways and exhibit activity in models of rheumatoid arthritis. Arthritis Rheum. 2008; 58(12): 376575 .

29. Park S, Park JA, Yoo H, et al. Proteasome inhibitor-induced cleavage of HSP 90 is mediated by ROS generation and caspase 10-activation in human leukemic cells. Redox Biol. 2017; 13: 470-476.

30. Altieri DC, Stein GS, Lian JB, et al. TRAP-1, the mitochondrial Hsp90. Biochim Biophys Acta. 2012; 1823(3): 767-73.

31. Felts SJ, Owen BA, Nguyen P, et al. The hsp90-related protein TRAP1 is a mitochondrial protein with distinct functional properties. J Biol Chem. 2000; 275(5): 3305-12.

32. Masgras I, Sanchez-Martin C, Colombo G, et al. The Chaperone TRAP1 As a Modulator of the Mitochondrial Adaptations in Cancer Cells. Front Oncol. 2017; 7: 58

33. Montesano Gesualdi N, Chirico G, Pirozzi G, et al. Tumor necrosis factor-associated protein 1 (TRAP-1) protects cells from oxidative stress and apoptosis. Stress. 2007; 10(4): 342-50.
34. Rondanin R, Lettini G, Oliva P, et al. New TRAP1 and Hsp90 chaperone inhibitors with cationic components: Preliminary studies on mitochondrial targeting. Bioorg Med Chem Lett. 2018; 28(13): 22892293.

35. Seo YH. Organelle-specific Hsp90 inhibitors. Arch Pharm Res. 2015 38(9): 1582-90.

36. Ohara PT, Vit JP, Bhargava A, et al. Gliopathic pain: when satellite glial cells go bad. Neuroscientist. 2009; 15(5): 450-63.

37. Lewis SS, Hutchinson MR, Rezvani N, et al. Evidence that intrathecal morphine-3-glucuronide may cause pain enhancement via toll-like receptor 4/MD-2 and interleukin-1beta. Neuroscience. 2010; 165(2): 569-83.

38. Ji RR, Chamessian A, and Zhang YQ. Pain regulation by non-neuronal cells and inflammation. Science. 2016; 354(6312): 572-577.

39. Lacagnina MJ, Watkins LR, and Grace PM. Toll-like receptors and their role in persistent pain. Pharmacol Ther. 2018; 184: 145-158.

40. Triantafilou M and Triantafilou K. Heat-shock protein 70 and heatshock protein 90 associate with Toll-like receptor 4 in response to bacterial lipopolysaccharide. Biochem Soc Trans. 2004; 32(Pt 4): 636-9.

\section{1. !!! INVALID CITATION !!!}

42. Gorska M, Popowska U, Sielicka-Dudzin A, et al. Geldanamycin and its derivatives as Hsp90 inhibitors. Front Biosci (Landmark Ed). 2012; 17: 2269-77.

43. Neef DW, Jaeger AM, and Thiele DJ. Heat shock transcription factor 1 as a therapeutic target in neurodegenerative diseases. Nat Rev Drug Discov. 2011; 10(12): 930-44.

44. Zou J, Guo Y, Guettouche T, et al. Repression of heat shock transcription factor HSF1 activation by HSP90 (HSP90 complex) that forms a stresssensitive complex with HSF1. Cell. 1998; 94(4): 471-80.

45. Powers MV, Clarke PA, and Workman P. Death by chaperone: HSP90, HSP70 or both? Cell Cycle. 2009; 8(4): 518-26.

46. Vlug AS, Teuling E, Haasdijk ED, et al. ATF3 expression precedes death of spinal motoneurons in amyotrophic lateral sclerosis-SOD1 transgenic mice and correlates with c-Jun phosphorylation, CHOP expression, somato-dendritic ubiquitination and Golgi fragmentation. Eur J Neurosci. 2005; 22(8): 1881-94.

47. Zhang L, Zhao H, Blagg BS, et al. C-terminal heat shock protein 90 inhibitor decreases hyperglycemia-induced oxidative stress and improves mitochondrial bioenergetics in sensory neurons. J Proteome Res. 2012; 11(4): 2581-93.

48. Kudryavtseva AV, Krasnov GS, Dmitriev AA, et al. Mitochondrial dysfunction and oxidative stress in aging and cancer. Oncotarget. 2016; 7(29): 44879-44905.

49. Jezek J, Cooper KF, and Strich R. Reactive Oxygen Species and Mitochondrial Dynamics: The Yin and Yang of Mitochondrial Dysfunction and Cancer Progression. Antioxidants (Basel). 2018; 7(1).

50. Im CN and Seo JS. Overexpression of tumor necrosis factor receptorassociated protein 1 (TRAP1), leads to mitochondrial aberrations in mouse fibroblast NIH/3T3 cells. BMB Rep. 2014; 47(5): 280-5. 